\title{
Zur Theorie der algebraischen Functionen.
}

Von

Adole Kreser in Breslau.

In den Art. 9 und 10 meiner Abhandlung: "Die Mouodromiegruppe einer algebraischen Gleichung bei linearen Transformationen der Variabelen*), ist implicite ein sllgemeiner, die algebraischen Functionen betreffender Satz bewiesen, welcher zusammen mit seiner schwieriger zu beweisenden Omkehrung ein Criterium ergiebt zur Entscheidung, ob eine gegebene algebraische Function von mehreren Variabeln als rationale Function mehrerer algebraischer Functionen von je einor Variabeln dargestellt werden kann oder nicht. Die Ableitung dieses Criteriums auf Grund einer erweiterten Darstellung jenes Satzes bildet das Ziel der vorliegenden Zeilen.

\section{$\S 1$.}

Die Grősse $x$ sei als algebraische Function der unabbängigen Variabeln $u, v_{1}, v_{3}, \ldots, v_{x}$ durch die Gleichung

$$
F\left(x, u, v_{1}, v_{2}, \ldots, v_{x}\right)=0
$$

definirt, welche bei Adjunction aller Constanten, d. h. aller von $u, v_{1}, v_{2}, \ldots, v_{x}$ nicht irrational abhängigen Grössen, oder kurz im Rationalitätsbereich $\Re_{0}$ irreducibel sein möge. Angenommen nan, eine beliebige Wurzel $x_{0}$ der Gleichung (1) könne in der Form

$$
x_{0}=\vartheta(y, z)
$$

dargestellt werden, wo $\vartheta$ eine rationale f'unetion mit Coefficienten aus dem Bereich $\Re_{0}, y$ eine algebraische Function von $u$ and $z$ eine algebraische Function von $v_{1}, v_{2}, \ldots, v_{x}$ bedeatet, so betrachte man die Gleichung (1) im Rationalitätsbereich aller algebraischen Functionen der Grössen $v_{\nu}$ oder kurz im Bereich $\Re_{1}$. Dann kann die linke Seite dieser Gleichung in Factoren zerfallen, welche nur noch in $x$ und $u$ rational und ganz sind; der im Bereich $\Re_{1}$ irreducibele Factor $F_{0}(x, u)$,

*) Diese Annalen Bd. XXVII, S. 126. 
welcher für $x=x_{0}$ verschwindet, besitze ausserdem die Wurzeln $x_{1}, x_{2}, \ldots, x_{m-1}$. Die algebraische Fonction $y$ wird nan durch eine Gleichnng

$$
f(y, u)=0
$$

definirt, welche bei Adjunction aller von $u$ nicht irrational abhängigen Grössen, also auch im Bereich $\Re_{1}$ irreducibel ist; da ferner in demselben Bereich auch die Gleichung

$$
F_{0}(x, u)=0
$$

irreducibel ist, so lehrt die Gleichung (2), dass die durch die Gleichung (4) definirte Gattung $x_{0}$ unter der durch die Gleichung (3) definirten Gattung $y$ im Rationalitätsbereich $\Re_{1}$ enthalten ist; die Ordnung der letzteren muss also nach bekannten Sätzen ein Vielfaches der Ordnung ron $x_{0}$, also etwa gleich $m m_{1}$ sein. Versteht man demnach unter $y_{1}, y_{2}, \ldots, y_{m m_{1}}$ die Wurzeln der Gleichung (3), so besteht bei passender Vertheilung der Indices, wenn man durch $\varphi$ einen rationalen Ausdruck mit Coefficienten aus dem Bereich $\Re_{1}$ bezeichnet, ein Gleichungssystem ron folgender Gestalt

(5) $x_{\lambda}=\varphi\left(y_{m_{1}+v}\right) ; \nu=1,2, \ldots, m_{1} ; \lambda=0,1, \ldots, m-1$.

Hieraus folgt sofort die Gleichung

$$
m_{1} x_{2}=\varphi\left(y_{2 m_{1}+1}\right)+\varphi\left(y_{\lambda m_{1}+2}\right)+\cdots+\varphi\left(y_{(\lambda+1) m_{1}}\right) ;
$$

also ist $x_{\lambda}$ nach dem Theorem von Lagrange rational im Bereich $\Re_{1}$ ausdrückbar durch jede symmetrische Function der Grössen $y_{\lambda m_{1}+1}$, $y_{2 m_{1}+2}, \ldots, y_{(2+1) m_{1}}$, welche keinem ihrer conjugirten Werthe gleich ist, z. B. durch das Product

$$
Y_{2}=\left(\alpha-y_{2 m_{1}+1}\right)\left(\alpha-y_{2 m_{1}+2}\right) \cdots\left(\alpha-y_{(\lambda+1) m_{1}}\right),
$$

in welchem $\alpha$ eine passend gewählte Constante bedeutet; $d$. h. es bestehen die Gleichungen

$$
x_{\lambda}=\psi\left(Y_{\lambda}\right),
$$

oder, was dasselbe bedeutet,

$$
x_{2}=\vartheta^{\prime}\left(Y_{2}, Z\right)
$$

wenn $\psi$ und $\vartheta^{\prime}$ rationale Ausdrücke sind, deren Coefficienten beziehentlich den Bereichen $\Re_{1}$ und $\Re_{0}$ angehören, und $Z$ eine algebraische Function der Grössen $v$ bedeutet.

Nun kann leicht gezeigt werden, dass die symmetrischen Functionen der Grössen $Y_{0}, Y_{1}, \ldots, Y_{m-1}$ dem Bereich $\Re_{1}$ angehören. Denn das mit der Unbestimmten $\beta$ gebildete Product

$$
Y_{2}^{\prime}=\left(\beta-\varphi\left(y_{2 m_{1}+1}\right)\right)\left(\beta-\varphi\left(y_{2 m_{1}+2}\right)\right) \cdots\left(\beta-\varphi\left(y_{(\lambda+1) m_{2}}\right)\right)
$$

hat mit keinem der Ausdrücke, welche aus ihm durch Umsetzung der

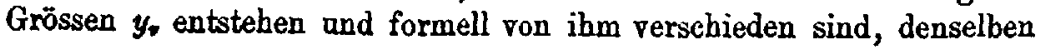


Werth, weil sonst zwischen den Grössen $\varphi\left(y_{0}\right)$ noch andere Gleichungen als die folgenden

$$
\varphi\left(y_{\lambda_{n_{2}+1}}\right)=\varphi\left(y_{2 m_{1}+2}\right)=\cdots=\varphi\left(y_{(2+1) m_{2}}\right)
$$

bestehen würden, was mit den Gleichungen (5) und der Irredacibilits̄t der Gleichung (4) unvereinbar ist. Demnach kann jede beliebige symmetrische Function der $m_{1}$ Grössen $y_{2 m_{2}+1}, y_{2 m_{2}+2}, \ldots, y_{(d+1)} m_{1}$, z. B. die Grösse $Y_{\lambda}$ im Bereich $\Re_{1}$ rational durch $Y_{2}^{\prime}$ ausgedrückt werden. Da nun offenbar die Gleichung

$$
Y_{\lambda^{\prime}}=\left(\beta-x_{2}\right)^{\mathrm{m}_{1}}
$$

besteht, so sina die symmetrischen Functionen von $Y_{0}^{\prime}, Y_{1}^{\prime}, \ldots, Y_{1 \mathrm{M}-1}^{\prime}$, mithin auch die symmetrischen functionen von $Y_{0}, Y_{1}, \ldots, Y_{m-1}$ im Bereich $\Re_{1}$ rational.

Die Gleichung ferner, deren Wurzeln die $m$ Grössen $Y_{0}, Y_{1}, \ldots, Y_{m-1}$ sind, ist im Bereich $\Re_{1}$ irreaucibel, weil sonst in Folge der Gleichung (6) auch die Gleichung (4) reducibel sein müsste, was der Definition von $F_{0}(x, u)$ widerspricht. Die beiden Grössen $x_{2}$ und $Y_{2}$ sind also algebraische Functionen $m^{\text {ter }}$ Ordnung ron $u$, welche im Bereich $\mathfrak{R}_{1}$ zu einer und derselben Gattung gehören, also auch dieselbe Gattungsdiscriminante*) besitzen; oder, was dasselbe bedeutet, der wesentliche Theiler der Discriminante**) der Gleichung

$$
\prod_{i=0}^{m-1}\left(Y-Y_{2}\right)=0
$$

muss mit dem wesentlichen Theiler der Discriminante der Gleichung (4) identisch sein; letzterer ist also, da die Grössen $Y_{2}$ Functionen von $u$ allein sind, von den Grössen $v$ unabbängig.

Dieselbe Schlussreihe wie für die Gleichung (4) kann nun für jede Gleichung $F_{1}(x, u)=0$ durchgeführt werden, in welcher links ein beliebiger, im Bereich $\Re_{1}$ irreducibeler Factor von $F\left(x, u, v_{1}, v_{2}, \ldots, v_{z}\right)$ steht. Um dies zu zeigen genügt der Nachweis, dass aus der Gleichung (2) eine Gleichung von derselben Form für jede Wurzel der Gleichung (1) folgt; denn der irreducibele Factor $F_{0}$ war nur dadurch ausgezeichnet, dass eine seiner Wurzeln $x_{0}$ der Gleichung (2) genügte. Dass non in der That jede Wurzel der Gleichung (1) einer Gleichung (2) genuggt, sieht man leicht, indem man eine Grösse $s$ einführt, durch welche man $y$ und $z$ im Bereich $\Re_{0}$ rational ansdrücken kann:

$$
\begin{aligned}
y & =\eta(s) ; z=\zeta(s), \\
x_{0} & =\vartheta(\eta(s), \xi(s)) .
\end{aligned}
$$

7) Vgl. Kronecker, Grundzüge § 8; Crelle's Joumal Bd. 92, S. 22.

*) Vgl. Kronecker, Ueber die Discriminante, 3 und 7, Crelle's Journal Bd. 91 , S. 313 und 329 . 
Ersetzt man hier die Grösse $s$ successive durch ihre sämmtlichen conjugirten Werthe, d. h. die Wurzeln der im Bereich $\Re_{0}$ irredncibeln Gleichnng, welcher die Grösse $s$ genügt, so erhält man auf der linken Seite der Gleichung (7) alle im Bereich $\Re_{0}$ mit $x_{0}$ conjugirten Grössen, also, wegen der Irreducibilität der Gleichung (1) im Bereich $\mathfrak{R}_{0}$, die sämmtlichen Wurzeln dieser Gleichung. Da nun auch die Grössen $\eta(s)$ und $\xi(s)$ in ihre conjugirten Werthe übergehen, also algebraische Functionen von $u$ bezw. den Grössen $v$ bleiben, so genügt jede Wurzel der Gleichung (1) einer Gleichung (2). Hiermit wird die an dem Polynom $F_{0}(x, u)$ entwickelte Schlussreihe auf das Polynom $F_{1}(x, u)$ äbertragbar; also hat die Discriminante der Gleichung $F_{1}(x, u)=0$ einen von den Grössen $v$ unabhängigen wesentlichen Theiler.

Berücksichtigt man nun noch, dass der wesentliche Theiler der Discriminante einer reducibeln Gleichung nichts anderes ist als das Product der wesentlichen Theiler der Discriminanten der irreducibeln Gleichungen, in welche die reducibele Gleichung sich auflöst*), so ergiebt sich das folgende Resultat:

Ist eine algebraische Function $x$ der Variabeln $u, v_{1}, v_{2}, \ldots, v_{x}$ rational ausdrüclibar durch eine algebraische Function von $u$ allein und eine algebraische Function der Grössen $v$ alltin, so muss, wenn man $x$ als algebraische Function von $u$ allein betrachtet und demgemäss alle algebraischen Functionen der Grössen $v$ adjungirt, die Discriminante der gegebenen die Function $x$ defmirenden Gleichung einen von den Grössen $v$ unabhängigen wesentlichen Theiler besitzen.

\section{$\S 2$.}

Eine Anwendung des erhaltenen Resultats führt zur Erledigung einer Frage, welche in der Abhandlung über die Monodromiegruppe ${ }^{* * *}$ ) aufgeworfen ist, der Frage nämlich, wann eine dem Bereich $\Re_{0}$ nicht angehörige rationale Function der Wurzeln der Gleichung (1) nach Adjunction einer algebraischen Function von $u$ allein rational werden kann.

Sind zunächst $y_{1}, y_{2}, \ldots, y_{r}$ die conjugirten Werthe irgend einer algebraischen Function von $u$, so constituirt die mit unbestimmten Coefficienten $\alpha$ gebildete Grösse

$$
\eta=\alpha_{1} y_{1}+\alpha_{2} y_{2}+\cdots+\alpha_{r} y_{r}
$$

eine Galois'sche Gattung, welche mit der Gattang

$$
\eta^{\prime}=\alpha_{1} y_{1}^{\prime}+\alpha_{1} y_{2}^{\prime}+\cdots+\alpha_{r} y_{r}^{\prime}
$$

identisch ist, wenn $y_{1}^{\prime}$ eine beliebige von den Unbestimmten $\alpha$ unabhäugige Grösse der Gattung $y_{1}$ und $y_{*}^{\prime}$ die entsprechend gebildete

*) Vgl. Kronecker, Veber die Discriminante, $\S 4$, a. a. O. S. 318.

*) Diese Annalen XXVIII, S, 130. 
Grösse der Gattung $y_{v}$ bedeutet. Ist also etwa $u-u_{0}$ ein Factor der Discriminante der Gattung $\eta$, so sind för $u=u_{0}$ zwei conjugirte Werthe von $\eta^{\prime}$, also auch zwei der Grössen $y_{v}^{\prime}$ einander gleich; also ist $u-u_{0}$ ein Factor der Discriminante der Grösse $y_{1}^{\prime} ;$ da nun $y_{1}^{\prime}$ eine beliebige von den Unbestmmaten $\alpha$ unabhängige Grōsse der Gattung $y_{1}$ bedentet, so ist $u-u_{0}$ auch ein Factor der Discriminante der Gattung $y_{1}$. Somit ergiebt sich das übrigens von selbst einleuchtende

Lemma. Versteht man unter $y_{1}, y_{2}, \ldots y_{r}$, die Wurceln einer Gleichung, deren Coefficienten ganze Functionen von $u$ sind, so ist die Diseriminante der Gattung $\alpha_{1} y_{1}+\alpha_{3} y_{2}+\cdots+\alpha_{r} y_{r}$, wo $\alpha_{1}, \alpha_{2}, \cdots, \alpha_{r}$ Onbestimmte sind, zusammengesetzt aus Linearfactoren, welche auch in der Discriminante der gegebenen Gleichung als Factoren des wesentlichen Theilers enthalten sind.

Jetzt sei speciell $y_{1}$ eine rationale Function der Wurceln $x_{0}, x_{1}, \ldots, x_{m-1}$ der Gleichung (1), welche nach Adjunction einer algebraischen Function ron $u$ allein rational wird; damn kann man das Resultat des $\$ 1$, auf die Function $y_{2}$ anwenden, bei welcher ein specieller Fall der Voraussetzung jenes Resultats stattfindet, und man kann schliessen, dass wenn man $y_{1}$ als algebraische Function von $u$ allein betrachtet, der wesentliche Theiler der Discriminante der Grösse $y_{1}$ von $v_{1}, v_{2}, \ldots, v_{x}$ unabhängig sein muss. Da nun nach einem Satze von Kronecker*) die Discriminante der Gattung $y_{1}$ ein Theiler der Discriminante der Gattong $\eta$, und diese ein Theiler der Discriminante der Gattung

$$
\xi=\alpha_{0} x_{0}+\alpha_{1} x_{1}+\cdots+\alpha_{n-1} x_{n-1}
$$

sein muss, weil die Gattung $y_{1}$ unter der Gattung $\eta$, und diese unter der Gattung $\xi$ enthalten ist, so folgt, dass die Discriminante der Gattung $\xi$ lineare Factoren besitzen muss, welche von den Grössen v unabhïngig sind. Nun ist nach dem eben bewiesenen Lemma jeder Linearfactor der Discriminante der Gattung $\xi$ zugleich ein Factor der Discriminante der Gattung $x_{0}$, wenn man $x_{0}$ als Function von $u$ allein betrachtet, also sicher ein Factor der Discriminante der Gleichung (1); wird also eine rationale Function der Wurseln der Gleichung (1) nach Adjunction einer algebraischen Function von $u$ allein rational, so muss die Discriminante dieser Gleichung Linearfactoren besitzen, welche von $v_{1}, v_{2}, \ldots, v_{x}$ unathängig sind. Ist dies durch die specielle Form der Gleichung (1) ausgeschlossen, wie z. B. wenn dieselbe die Gestalt $F(x, u x+v)=0$ besitat, unter $F(x, y)$ ein im Bereich $\Re_{0}$ irreducibeles Polynom verstanden, so kann keine rationale Function der Wurzeln dieser Gleichung nach Adjunction einer algebraischen Function von $u$ allein rational werden. Damit ist der zweite der beiden Fälle,

*) Vgi. Grundzüge § 9, Crelle's Journal Bd. 92, S. 26. 
welche am Ende des Art. 8 der citirten Abhandlung über die Monodromiegruppe bezeichnet sind, als unmöglich nacbgewiesen.

\section{§3.}

Bemerkenswerth ist nun, dass das Resultat des $\S 1$ sich umkehren lässt. Dem Beweis dieser Behauptung müssen aber zwei einfache Hülfsbetrachtungen vorausgeschickt werden.

(A) Durch die Gleichung

$$
G(x, u)=0,
$$

deren Coefficienten von den Unbestimmten $v_{1}, v_{2}, \ldots, v_{x}$ in beliebiger Weise algebraisch abhängig sein mögen, sei $x$ als algebraische Function von $u$ definirt; ersetzt man $v_{v}$ durch die Constante $c_{v}$, so gehe die Gleichung (8) in die Gleichung

$$
G^{\prime}(x, u)=0
$$

über. Dann kann leicht gezeigt werden, dass bei passender Wahl der Constanten $c_{v}$ der wesentliche Theiler der Discriminante der Gleichung (9) aus dem wesentlichen Theiler der Discriminante der Gleichung (8) erhalten wird, indem man $v$, durch $c_{v}$ ersetzt, und dass die Werthsysteme $c_{\nu}$, bei denen dies nicht stattfindet, höchstens eine algebraische Mannichfaltigkeit von $x-1$ Dimensionen erfüllen können.

Setzt man nämlich:

$$
\begin{aligned}
G(x, u) & =U_{0} x^{m_{t}}+U_{1} x^{m-1}+\cdots+U_{m}, \\
G^{\prime}(x, u) & =U_{0}^{\prime} x^{m}+U_{1}^{\prime} x^{m-1}+\cdots+U_{m}^{\prime}, \\
H(x, u) & =x^{m}+U_{1} x^{m-1}+U_{2} U_{0} x^{m-2}+\cdots+U_{m} U_{0}^{m-1}, \\
H^{\prime}(x, u) & =x^{m}+U_{1}^{\prime} x^{m-1}+U_{2}^{\prime} U_{0}^{\prime} x^{m-2}+\cdots+U_{m}^{\prime} U_{0}^{\prime}{ }^{m-1}, \\
\frac{\partial H(x, u)}{\partial x} & =H_{1}(x, u), \quad \frac{\partial H(x, u)}{\partial u}=H_{2}(x, u),
\end{aligned}
$$

indem unter $V_{0}, D_{1}, \ldots$ ganze Functionen von $u$, unter $U_{0}^{\prime}, V_{1}^{\prime}, \ldots$ dieselben ganzen Functionen nach Ersetzung von $v_{v}$ durch $c_{\text {, verstanden }}$ werden, so sind die wesentlichen Theiler der Discriminanten der Gleichungen (8) und (9) beziehentlich identiseh mit den wesentlichen Theilern der Discriminanten der Gleichungen *)

$$
\begin{aligned}
H(x, u) & =0, \\
H^{\prime}(x, u) & =0 .
\end{aligned}
$$

Nun kann der ausserwesentliche Theiler der Discriminante der Gleichung (10) definirt werden als der grösste gemeinsame Theiler der Coefficienten in der homogenen Function

*) Vgl. Kronecker, Ueber die Discriminante $\$ 7$, a. a. 0. S. 329. 


$$
P(U, V)=\prod_{v=0}^{m-1}\left(U B_{1}\left(\xi_{\nu}, u\right)+\nabla H_{2}\left(\xi_{n}, u\right)\right)
$$

der Unbestimmten $V$ und $V$, wenn $\xi_{0}, \xi_{1}, \ldots, \xi_{m-1}$ die Wurzeln der Gleichung (10) sind*). Haben aber allgemein mehrere gauke Functionen von $u$, deren Coefficienten algebraisch von den Grössen $v$ abhängen, z. B. $g_{1}(u ; v), g_{2}(u ; v), \ldots$ einen grössten gemeinsamen Theiler $T(u ; v)$, so müssen gewisse ganze Functionen der Coefficienten von $u$ in den Polynomen $g_{v}(u ; v)$ verschwinden, während eine be stimmte solche Function von Null verschieden bleibt, sodass für unbestimmte Werthe der Grössen $v$ ein System von Relationen

$$
\left|h_{0}(v)\right|>0, \quad h_{1}(v)=h_{2}(v)=\cdots=0
$$

besteht, in welchen $h_{0}, h_{1}, \ldots$ gewisse algebraische Functionen der Grössen $v$ bedeuten; und diese Relationen können so gewählt werden, dass umgekehrt aus ihrem Bestehen die Existenz eines grössten gemeinsamen Theilers der Functionen $g_{v}(u ; v)$ geschlossen werden hann, dessen Grad in $u$ derselbe ist wie der Grad von $T(u ; v)$. Giebt man also den Unbestimmten $v$, constante Werthe $c_{\nu}$, für welcbe die Grösse $h_{0}(c)$ von Null verschieden ist, so haben die Polynome $g_{v}(u ; c)$ sicher den gemeinsamen Theiler $T(u ; c)$ und einen grössten gemeinsamen Theiler, dessen Grad in $u$ derselbe ist wie der Grad von $T(u ; v)$, der sich also von $T(u ; c)$ böchstens um einen constanteu Factor unterscheiden kann. Sollen dagegen die Polynome $g_{r}(u ; c)$ nach Division durch $T(u ; c)$ noch eine nicht constante ganze Function von $u$ als gemeinsamen Theiler besitzen, so muss $h_{0}(c)=0$ sein, also eine Gleichung bestehen, welche nur für eine höchstens $(x-1)$-fache Mannichfaltigkeit von Werthsystemen $c$ erfüllt sein kann, weil $h_{0}(v)$ für unbestimmte Werthe der Grössen $v$ von Null verschieden ist. Versteht man also speciell unter $g_{1}(u ; v), g_{2}(u ; v), \ldots$ die Coefficienten in $P(U, V)$, so ergiebt sich, dass abgesehen von einer höchstens $(x-1)$-fachen algebraischen Mannichfaltigkeit von Werthsystemen $c$ der ausserwesentliche Theiler der Discriminante der Gleichung (11) aus dem ausserwesentlichen Theiler der Discriminante der Gleichung (10) erbalten wird, indem man $v_{r}$ durch $c_{r}$ ersetzt. In derselben Weise erhält man also den wesentlichen Theiler der Discriminante der Gleichung (11), oder was dasselbe ist, der Gleichung (9), aus dem wesentlichen Theiler der Discriminante der Gleichung (10), oder was dasselbe ist, der Gleichung (8), womit die aufgestellte Behauptung bewiesen ist.

(B) Ein zweiter vorauszuschickender Hülfssatz, dessen Richtigkeit übrigens fast von selbst einleachtet, ist der folgende. Fine im

*) Kronecker, 2. a. 0. \$ 8, \$. 334.* 
Bereich $\Re_{1}$ irreducibele ganze Function $G(x, u ; v)$ von zwei oder mehr Variabeln $x, u_{1}, u_{2}, \ldots$, deren Coefficienten von den Unbestimmten $v_{1}, v_{2}, \ldots, v_{x}$ algebraisch abbängen, kann, wenn die Unbestimmte $v_{\nu}$ durch die Constante $c_{v}$ ersetzt wird, höchstens für eine $(x-1)$-fache algebraische Mannichfaltigkeit von Werthsystemen $c$ in Factoren, welche ganze Functionen von $x, u_{1}, u_{2}, \ldots$ sind, zerlegbar werden.

Denn bezeichnet man durch $\eta_{0}, \eta_{1}, \ldots, \eta_{m-1}$ die Wurzeln der Gleichung $G(x, u ; v)=0$ mit der Unbekannten $x$, so besteht für die Existenz einer in $G(x, u ; c)$ aufgehenden ganzen Function von $x, u_{1}, u_{2}, \ldots$, welche in $x$ vom Grade $p<m$ ist, eine hinreichende und nothwendige Bedingung in Folgendem.

Es müssen die symmetrischen Functionen eines gewissen Systems von $p$ Worzeln $\eta_{i_{1}}, \eta_{i_{2}}, \ldots, \eta_{i p}$ für $v_{\nu}=c_{\nu}$ rationale Functionen der Grössen $u_{1}, u_{2}, \ldots$ werden; oder es muss, wenn man wiederum

$$
G(x, u ; v)=U_{0} x^{m}+U_{1} x^{n-1}+\cdots
$$

setzt und unter $J_{0}, D_{1}, \ldots$ ganze Functionen der Grössen $u$ versteht, das mit der Unbestimmten $\alpha$ gebildete Product

$$
U_{0}{ }^{\mu}\left(\alpha-\eta_{i_{1}}\right)\left(\alpha-\eta_{i_{2}}\right) \cdots\left(\alpha-\eta_{i_{p}}\right)=X,
$$

durch welches alle jene symmetrischen Functionen rational ausdrückbar sind, und welches stets eine ganze algebraische Function der Grössen $u$ ist, für $v_{y}=c_{v}$ in eine ganze rationale Function der Grössen $u$ übergehen. Nun kann man aus den Dimensionszahlen der Coefficienten $U_{\nu}$ bezüglich der Variabeln $u$ leicht eine obere Grenze $\gamma$ bestimmen, welche die Dimension ron $X$, wenn diese Grösse eine ganze rationale Function wird, nicht überschreiten kann. Denn setzt man für $v_{\nu}=c_{\nu}$

$$
X=g\left(\alpha, u_{1}, u_{2} \ldots\right)
$$

so ist offenbar die Grösse

$$
g\left(-\frac{\beta}{U_{0}}, u_{1}, u_{2}, \cdots\right)=\left(\beta-U_{0} \eta_{i_{1}}\right)\left(\beta-U_{0} \eta_{i_{z}}\right) \cdots\left(\beta-U_{0} n_{i_{p}}\right)
$$

ein Factor von

$$
U_{0}^{m-1} G\left(\frac{\beta}{U_{0}}, u ; c\right)=\left(\beta-U_{0} \eta_{1}\right)\left(\beta-D_{0} \eta_{1}\right) \cdots\left(\beta-U_{0} \eta_{\mu-1}\right),
$$

wenn in $U_{0}$ äberall $v_{\nu}=c_{\nu}$ gesetzt wird; ist also $\gamma$ die Dimension des Polyuoms $U_{0}^{m-1} G(x, u ; v)$ in den Grössen $u$, so kann die Dimension der ganzen Function $g\left(x, u_{1}, u_{2}, \ldots\right)$ den Werth $\gamma$ sicher nicht übersteigen. Demnach bestehen, wenn die Grösse $X$ für $v_{\nu}=c_{\nu}$ in den Grössen $u$ rational wird, die Differentialgleichungen

$$
\frac{\partial^{\gamma+1} X}{\partial u_{1}^{\gamma_{2}} \partial u_{2}^{\gamma_{2}} \cdots}=0
$$

worin unter $\gamma_{1}, \gamma_{2}, \ldots$ irgend welche Zahlen der Reihe $0,1, \ldots, \gamma+1$ zu verstehen sind, welche der Gleichung 


$$
1+\gamma=\gamma_{1}+\gamma_{2}+\cdots
$$

genügen. Versteht man nun unter $X^{\prime}, X^{\prime \prime}, \ldots$ dio sämmtlichen Producte von derselben Gestalt wie $X$, so darf für unbestinumte Werthe der Grössen $v$ keine der über alle möglichen Systeme $\gamma_{1}, \gamma_{2}, \ldots$ erstreckten Summen

$$
\sum_{(y)} V_{\gamma_{1} \gamma_{2} \ldots} \frac{\hat{\partial}^{\gamma+1} X^{(y)}}{\partial u_{1}{ }^{\gamma_{1}} \partial u_{2}{ }^{\gamma_{1}} \cdots}
$$

bei unbestimmten Coefficienten $V$ verschwinden, weil sonst eine der Grössen $X^{(\boldsymbol{})}$ eine ganze rationale Fuuction der Grössen $u$ wäre, also $G(x, u ; v)$ für unbestimmte Werthe der Grössen $v$ reducibel wäre, was der Voraussetzung widerspricht. $\mathrm{Da}$ man nun, unter $\Psi$ rationale Ausdrücke verstanden, setzen kann

$$
\frac{\partial^{\gamma+1} X^{(v)}}{\partial u_{1}^{\gamma_{1}} \partial u_{2}^{\gamma_{2}} \ldots}=\Psi_{\gamma_{1} y_{2} \ldots}\left(X^{(v)}\right),
$$

so besitzt man in dem Product

$$
\equiv=\prod \sum_{y_{1} \gamma_{2} \ldots} V_{y_{1} y_{2} \ldots} \Psi_{y_{1} y_{2} \ldots}\left(X^{(v)}\right)
$$

einen rationalen Ausdruck, welcher für unbestimmte Werthe der Grössen $v_{\nu}$ sicher von Null verschieden ist, und welcher für $v_{\nu}=c_{v}$ verschwinden muss, wenn das Polynom $G(x, u ; c)$ einen Factor haben soll, der in $x ; u_{1}, u_{2}, \ldots$ rational und ganz, und in $x$ rom Grade $p$ ist. Setzt man also in der Gleichung

$$
\equiv=0
$$

die Werthe $v_{\gamma}=c_{\gamma}$ ein, so erhält man für die Grössen $c$ ein System von Bedingungsgleichungen, welches sicher nicht identisch erfullt ist, also höchstens eine $(x-1)$-fache algebraische Mannichfaltigkeit im Gebiet der Grössen $c$ definiren kann, womit die aufgestellte Behauptung bewiesen ist.

\section{$\$ 4$.}

Um jetzt nach Erledigung der einfachen Vorfragen zum Beweis der Behauptung zu kommen, dass das Resultat des $\$ 1$ sich umkehren lässt, diene als Ausgangspunkt die Bemerkung, dass es nur eine endliche Anzahl von Gattungen $n$-werthiger algebraischer Functionen von einer Variabeln $u$ geben kann, deren Gattangsdiscriminante eine bestimmte ganze rationale Function von $u$ ist. Der algebraische Beweis dieser Behauptung scheint ziemlich schwierig zu sein; die Richtigkeit derselben ergiebt sich aber leicht aus der Betrachtung der $n$-blättrigen Riemann'schen Flächen*).

") Vgl. F. Klein, Ceber Riemann's Theorie der algebraischen Fanctionen, $\S 19$, S. 64 
Zunächst ist klar, dass eine algebraische Function sich nur an den Nollstellen ibrer Gattnngsdiscriminante verzweigen kann, da für jeden von diesen Nullstellen verschiedenen Werth $u_{0}$ der unabhängigen Variabeln*) eine Grösse der Gattung gefunden werden kann, welche für $u=u_{0}$ lauter von einander versehiedene conjugirte Werthe besitzt, sich also unmöglich an der Stelle $u=u_{0}$ verzweigen kann. Da nun jede Riemann'sche Fläche eine bestimmte Gattung algebraischer Functionen von $u$ definirt, so wird die endliche Anzahl der Gattungen mit gegebener Gattungsdiscriminante erwiesen sein, wenn man gezeigt hat, dass nur eine endliche Anzahl verschiedener, $d . h$. nicht conform aufeinander abbildbarer Riemann'scher Flächen existirt, welche sich an keinen andern Punkten der $u$-Ebene als den gegebenen $P_{1}, P_{2}, \ldots, P_{k}$ verzweigen. Diese Behauptung kann aber leicht bewiesen werden. Zieht man nämlich eine sich selbst nicht schneidende Linie $L$ von $P_{1}$ nach $P_{2}$, von $P_{2}$ nach $P_{3}$, u. s. f., von $P_{l}$ ins Unendliche, welches durch $P_{h+1}$ bezeichnet werden möge, so ist jeder einzelne $Z$ weig einer nur in den Punkten $P$ verzweigten algebraischen Function in der ganzen Ebene mit Ausnahme der Linie $L$ eindeutig bestimmt, solange die Linie $L$ nicht überschritten wird, und die zugehörige Riemann'sche Fläche ist völlig gegeben, sobald man weiss, in welcher Weise bei Ueberschreitung der Linien $L$ in einer ein für alle Mal festzusetzenden Richtung an jeder der Strecken $P_{v} P_{v+1}$ die $n Z$ Weige ineinander übergehen. Bezeichnet man dieselben durch $1,2, \ldots, n$, so gehört zu jeder Strecke $P_{\nu} P_{++1}$ eine bestimmte den Zusammenhang der Zweige längs dieser Strecke definirende Substitution

$$
S_{v}=\left(\begin{array}{cc}
1,2, \ldots, n \\
v_{1}, v_{2}, \ldots, & v_{n}
\end{array}\right)
$$

Gebört za jeder Strecke $P_{v} P_{r+1}$ bei einer algebraischen Function dieselbe Substitution $S_{\nu}$ wie bei einer zweiten, so sind die zugehörigen Riemann'schen Flächen identisch; dasselbe findet übrigens offenbar schon dann statt, wenn die bei der einen Function auftretenden Substitutionen $S_{\nu}$ durch Transformation mit einer für alle Indices $\nu$ sich gleich bleibenden Substitution aus den bei der andern auftretenden ableitbar sind. Da es nun aber nur eine endliche Anzahl von Umsetzungen der Zahlen 1, 2, ..,n giebt, und aus diesen für eine endliche Anzahl von Strecken $P_{v} P_{v+1}$ die zugehörigen Substitutionen $S_{v}$ auszuwählen sind, so ist klar, dass nur eine endliche Anzahl wesentlich verschiedener $n$-blättriger Riemann'scher Flächen existiren kann, welche sich an keinen andern Stellen als den Stellen $P_{v}$ verzweigen. $Z u$ einer gegebenen Gattungsdiscriminante gehört also nur eine endliche Anzahl von $\boldsymbol{n}$-werthigen Gattungen algebraischer Functionen von $u$.

-) VgL Kronectier a. a. O. \$5, S. 322. 
Jetzt werde durch die in Bereich $\mathfrak{R}_{1}$ irreducibele Gleichung

$$
F_{0}(x, u ; v)=0 \text {, }
$$

deren linke Seite von den Parametern $v_{1}, v_{2}, \ldots, v_{x}$ algebraisch abhängen möge, die Grösse $x$ als eine algebraische Function von $u$ dofinirt, deren Gattnngsdiscriminante von den Grössen $v_{v}$ unabhängig ist. Dann kann man die letzteren nach $\$ 3$ (A) durch derartige Constanten $c$, ersetzen, dass der wesentliche Theiler der Discriminanto der Gleichung

$$
F_{0}\left(x^{\prime}, u ; c\right)=0
$$

aus demjenigen der Gleichung (12) erhalten wird, indem man $v$, durch $c$, ersetzt; da nun letzterer nach Voraussetzung von den Grössen v unabhängig ist, so haben die Discriminanten der Gleichungen (12) und (13) denselben wesentlicben Theiler; dabei können nach \$3 (A) diejenigen Werthsysteme $c$, für wolche dies nicht stattfindet, böchstens eine $(x-1)$-fache algebraische Mannichfaltigkeit $\mathfrak{M}$ erfüllen. Somit bann wan durch Gleichungen (13) unendlich viele functionen $x^{\prime}$ definiren, welche eine und dieselbe Gattungsdiscriminante besitzen, sich also auf eive endliche Anzahl von Gattungen vertheilen mussen; schliesst man ausser der Mannichfaltigkeit $\mathfrak{D}$ auch diejenigen Werthsysteme $c$ aus, für welche die Gleichung (13) reducibel wird und welche nach $\$ 3$ (B) ebenfalls nur eine höchstens $(x-1)$-fache algebraische Mannichfaltigkeit $\mathfrak{M}^{\prime}$ erfüllen können, so mögen sich alle durch die Gleichungen (13) definirten algebraischen Functionen $x^{\prime}$ auf die $p$ Gattungen vertheilen, welche durch die Gleichungen

$$
F_{0}\left(x^{(v)}, u ; C^{(v)}\right)=0, \quad v=1,2, \ldots, p
$$

definirt werden. Dann lässt sich zeigen, dass alle diese Gattungen identisch sind.

Zum Beweis dieser Behauptung soll indirect bewiesen werden, dass eine Wurzel $x$ der Gleichung (12) durch eine der Wurzeln $z(\beta)$ der Gleichungen (14) rational ansdrückbar ist. Wäre dies nicht der Fall, so könnten in der auf den Bereich $\Re_{1}$ bezogenen Gruppe der reducibeln Gleichung

$$
F_{0}(x, u ; v) \prod_{v=1}^{p} F_{0}\left(x, u ; C^{(v)}\right)=0
$$

unter den Substitutionen, welche eine der Wurzeln $x$ des ersten Factors am Platze lassen, niemals die sämmtlichen Substitutionen vorkommen, welche eine der Grössen $x^{(p)}$, also eine der Warzeln der $p$ übrigen Factoren am Platze lassen. Dagegen giebt es, wenn das Werthsystem $c_{\text {v }}$ den Mannichfaltigkeiten $\mathfrak{M}$ and $\mathfrak{Q}^{\prime}$ nicht angehört, in der Gleichung 


$$
F_{0}(x, u ; c) \prod_{v=1}^{p} F_{0}\left(x, u ; C^{(v)}\right)=0
$$

zu jeder Wurzel $x^{\prime}$ des ersten Factors eine Wurzel $x$ te eines der $p$ letzten Factoren, durch welche jene Wurzel $x^{\prime}$ rational ausdrückbar ist; in der anf den Bereich $\Re_{1}$ bezogenen Gruppe der Gleichung (16) kommt also unter den Substitutionen, welche eine Wurzel $x^{\prime}$ am Platze lassen, stets ein vollständiges System aller derjenigen Substitutionen vor, welche eine gewisse Wurzel $x^{(\rho)}$ am Platze lassen. Die Gruppen der Gleichungen (15) und (16) sind demnach verschieden.

Ist nun etwa $\Gamma(\xi ; v)$ ein irreducibeler Factor der Galois'schen Resolvente der Gleichung (15), so ist offenbar $\Gamma(\xi ; c)$ ein rationaler Factor der Galois'schen Resolvente der Gleichung (16); die Gruppe der letzteren ist also in der Gruppe der Gleichung (15) enthalten oder mit ihr identisch. Letzteres kann, wie soeben gezeigt ist, nicht eintreten; also muss für jedes der Werthsysteme $c$, welche den Mannichfaltigkeiten $\mathfrak{M}$ und $\mathfrak{M R}^{\prime}$ nicht angehören, das Polynom $r(\xi ; c)$ reducibel sein, während bei unbestimmten Werthen $v_{y}$ das Polynom $\Gamma(\xi ; v)$ irreducibel ist. Dies Resultat würde aber nach $\S 3(\mathrm{~B})$ unmöglich sein, weil höchstens eine $(x-1)$-fache algebraische Mannichfaltigkeit von Werthsystemen $c$ existiren kann, fur welche das irreducibele Polynom $\Gamma(\xi ; v)$ bei Ersetzung von $v_{v}$ durch $c_{\nu}$ reducibel wird. Die Annahme, von welcher man ausgegangen ist, dass nämlich keine Wurzel der Gleichung (12) durch eine der Wurzeln der Gleichungen (14) rational ausdrückbar sei, ist also unrichtig, und es muss demnach für eine Wurzel $x$ der Gleichung (12) eine Gleichung von folgender Gestalt bestehen:

$$
x=\varphi(x(s), u ; v),
$$

in welcher die rechte Seite ron $x^{(\rho)}$ und $u$ rational, von den Grössen $v$ algebraisch abhängt. Da nun im Bereich $\Re_{1}$ die Grössen $x$ und $x(0)$ Wurzeln irreducibeler Gleichungen desselben Grades sind, so besteht, wenn unter $\psi$ ein ähnlicher Ausdruck wie $\varphi$ verstanden wird, anch eine Gleichung

$$
x(\rho)=\psi(x, u ; v)
$$

aus den Gleichungen (17) und (18) aber folgt

also auch

$$
F_{0}(\varphi(x(e), u ; v), u ; v)=0
$$

ebenso andrerseits

$$
F_{0}(\varphi(x(\rho), u ; c), u ; c)=0 \text {; }
$$

$$
F_{0}\left(\psi(x, u ; v), u ; C^{(\varrho)}\right)=0,
$$

also, $\mathrm{ds} x$ in $x^{\prime}$ übergeht, wenn $v_{v}$ durch $c_{v}$ ersetzt wird

$$
F_{0}\left(\psi\left(x^{\prime}, u ; c\right), u ; C^{(\varphi)}\right)=0 \text {. }
$$


Somit ergiebt sich das Resultat, dass die durch (13) definirten Functionen $x^{\prime}$ sich nicht nur auf eine endliche Anzahl von Gattangen vertheilen, sondern sogar alle einer und derselben Gattung angehöran. Hierans Hiesst sofort der folgende

\section{Lehrsatz I.}

Sind die Coefficienten der eine algebraische Function von einer Variabeln definirenden Gleichung, nicht aber die Verzweigungspunkte dieser Function von gewissen $x$ Parametern algebraisch alhängig, sa gehören alle für specielle Werthe der Parameter erhaltenen algbbraischen Functionen, abgesehen höchstens von einer $(x-1)$-fachen algebraischen Mannichfaltigkeit im Gebiet der $x$ Parameter, einer und derselben Gattung an.*)

Ein weiteres Resultat, welches aus der Gleichung (18) abgelesen werden kann, ist dieses, dass die von den $x+1$ Variabeln $u, v_{4}, \cdots, v_{x}$ abhängige Grösse $x$ durch eine algebraische Function von $u$ allein, nämlich $x^{(\rho)}$, und die in den Coefficienten des Ausdruckes $\varphi$ auftretenden algebraischen Functionen der Grössen $v$, welche alle durch eine einzige algebraische Function dieser Grössen ausgedrückt werden können, also auch durch eine algebraische Function von $u$ allein und eipe algebraische Function der Grössen $v$ allein im Bereich $\Re_{0}$ rational ausdrückbar ist. Damit ist die Dmkehrung des in $\$ 1$ erhaltenen Resultats erwiesen und es exgiebt sich der folgende

\section{Lehrsatz II.}

Eine algebraische Function der Variabeln $u, v_{1}, v_{2}, \ldots, v_{x}$ ist dann und nur dann rational ausdrïckbas durch eine abetraische Function von u allein und eine algebraische Function der Grössen v allein, wenn bei Betrachtung von $x$ als algebraischer Function von $u$ allein und dementsprecherder Adjunction aller algebraischer Functionen der Grössen $v$ die Discriminante der gegebenen, die Function $x$ definirenden Gleichung einen von den Grössen v unabhängigen wesentlichen Theiler besitat.

Hieraus kann nun leicht ein Kriterium dafü abgeleitet perden, ob eine algebraische Function von mehreren Variabeln rational dureh algebraische Functionen von je einer dieser Variabeln ansgedrückt werden kann oder nicht.

Die algebraische Function $x$ der $h_{b}$ Variabeln $u_{1}, u_{2}, \ldots, u_{k}$ sei für jeden Werth von $v$ rational durch eine algebraische Function von $u_{v}$ allein und eine algebraische Function der übrigen $h-1$ Grössen $u$ rational ausdrückbar, sodass man setzen kann

$$
\begin{array}{rlr}
x & =\vartheta_{1}\left(\sigma_{1}\left(u_{2}, u_{3}, \cdots, u_{k}\right), \quad \tau_{1}\left(u_{1}\right)\right) \\
& =v_{2}\left(\sigma_{2}\left(u_{1}, u_{3}, \cdots, u_{k}\right), \quad \tau_{3}\left(u_{2}\right)\right)=\cdots \\
& =v_{3}\left(\sigma_{3}\left(u_{1}, u_{2}, \cdots, u_{k-1}\right), \tau_{4}\left(u_{k}\right)\right)
\end{array}
$$

*) In der Riemann'schen Terminologie einer und derselben "Clewe“. 
wobei unter $\vartheta_{1}, \vartheta_{2}, \cdots, \vartheta_{4}$ rationale Ausdrücke, deren Coefficienten von den Grössen $u$ nicht irrational abhängig sind, und unter $\sigma, \tau$ algebraische Functionen der beigefügten Argumente zu verstehen sind. Dann kann man annehmen, dass die Ordnung der algebraischen Function $\sigma_{1}$ dieselbe ist wie die Ordnung der Grösse $x$ nach Adjunction aller algebraischen Functionen von $u_{1}$, d. h. gleich dem Grade der nach dieser Adjunction irreducibeln Gleichung, welcher die Grösse $x$ genügt. Denn sind die Wurzeln dieser Gleichung $x_{0}=x, x_{1}, x_{2}, \cdots, x_{n-1}$, so bestände andernfalls das Gleichungssystem

$$
\begin{aligned}
& x_{0}=\vartheta_{1}\left(\sigma_{1 v}, x_{1}\left(u_{1}\right)\right) \text {, } \\
& x_{1}=\vartheta_{1}\left(\sigma_{1, n_{1}+y}, \tau_{1}\left(u_{1}\right)\right) \text {, } \\
& x_{n-1}=\vartheta_{1}\left(\sigma_{1,(n-1) n_{1}+v}, \tau_{1}\left(u_{1}\right)\right) \quad v=1,2, \cdots, n_{1} \\
& r=n n_{1} \text {, }
\end{aligned}
$$

wo unter $\sigma_{11}=\sigma_{1}\left(u_{2}, u_{3}, \cdots, u_{h}\right), \sigma_{12}, \sigma_{13}, \cdots, \sigma_{1}$, die sämmtlichen $r$ conjugirten Werthe der algebraischen Function $\sigma_{1}$ zu verstehen sind. Diese conjugirten Werthe sind dieselben, gleichviel ob man den Rationalitätsbereich aller Constanten oder denjenigen aller algebraischen Functionen von $u_{1}$ zu Grunde legt; denn die nach Adjunction aller Constanten irreducibele Gleichung, welcher die Grösse $\sigma_{1}\left(u_{2}, u_{3}, \cdots, u_{k}\right)$ genügt, kann offenbar durch Adjunction algebraischer Functionen von $u_{1}$ nicht reducibel werden. Aus den Gleichungen (20) und der Verschiedenheit der Werthe $x_{y}$ folgt, dass der mit der Unbestimmten $\alpha$ gebildete Ausdruck

$$
s_{\lambda}=\prod_{\nu=1}^{n_{1}}\left\{\alpha-\vartheta_{1}\left(\sigma_{1, \lambda n_{1}+\nu}, \tau_{1}\left(u_{1}\right)\right)\right\} \quad \lambda=0,1, \cdots, n-1
$$

keinem formell von ihm verschiedenen Ausdrucke, welcher aus ihm durch Omsetzung der $r$ Grössen $\sigma_{11}, \sigma_{12}, \cdots, \sigma_{1}$, entsteht, dem Werthe nach gleich sein kann; durch $s_{2}$ sind also alle symmetrischen Functionen der Grössen

$$
\sigma_{1, \lambda n_{1}+1}, \sigma_{1, \lambda n_{1}+2}, \cdots, \sigma_{1,(2+1) \pi_{1}}
$$

rational nach dem bekannten Theorem von Lagrange ausdrückbar; da nun aber offenbar die Gleichung

$$
s_{2}=\left(\alpha-x_{2}\right)^{n_{1}}
$$

besteht, so folgt, wenn man

$$
S_{2}=\prod_{\nu=1}^{n_{1}}\left(\alpha-\sigma_{1,2 n_{1}+1}\right)
$$

setzt, dass die symmetrischen Functionen der Grössen $S_{0}, S_{1}, \cdots, S_{*-1}$ 
nach Adjunction algebraischèr Functionen von $u_{1}$ rational sind; da nun aber die Grössen $S_{2}$ ron $u_{1}$ unabbängig sind, so sind die genannten symmetrischen Functionen nach alleiniger Adjunction von Constanten rational, d. h. sie sind rationale Functionen von $t_{2}, u_{3}, \cdots, u_{4}$. Weil ferner die Grösse $S_{2}$ keiper formell von ihr verschiedenen Grösse, welche durch Umsetzung der Grössen $\sigma_{11}, \sigma_{12}, \cdots, \tau_{1 r}$ aus ihr entsteht, dem Werthe nach gleich ist, so bann durch $S_{2}$ jede symmetrische Function der Grössen (21), also auch die Grösse

$$
x_{2}=\frac{1}{n_{i}} \sum_{v=1}^{n_{1}} \vartheta_{1}\left(\sigma_{1,2 w_{i}+1}, \tau_{1}\left(u_{1}\right)\right)
$$

ratioual ausdrückbar, speciell kann man setzen

$$
x_{0}=x=\theta\left(S_{0}, \tau_{1}\left(u_{1}\right)\right) \text {, }
$$

wenn unter $\theta$ ein rationaler Ausdruck derselben Art wie $\vartheta_{1}$ verstanden wird. $\mathrm{Da}$ nun $S_{0}$ eine $n$-werthige algebraische Function von $u_{2}, u_{3}, \cdots, u_{2}$ ist, so ist bewiesen, dass man ohne Beschränkung der Allgemeinheit annehmen kann, in der Gleichung:

$$
x=\vartheta_{1}\left(\sigma_{1}\left(u_{2}, u_{3}, \cdots, u_{k}\right), \tau_{1}\left(u_{1}\right)\right)
$$

sei $\sigma_{1}$ eine $n$-werthige algebraische Function, wenn die Grösse $x$ bei Adjunction aller algebraischen Functionen von $u_{1}$ einer irreducibeln Gleichung $n^{\text {ten }}$ Grades genügt. Dann sind $x$ und $\sigma_{1}$ algebraische Grössen derselben Ordnung, also folgt aus der rationalen Ausdrückbarkeit von $x$ durch $\sigma_{1}$ auch umgekehrt, dass man setzen kann

$$
\sigma_{1}\left(u_{2}, u_{3}, \cdots, u_{h}\right)=v_{0}\left(x, \tau_{0}\left(u_{1}\right), u_{1}, u_{2}, \cdots, u_{4}\right) \text {, }
$$

wobei $\vartheta_{0}$ einen rationalen Ausdruck mit constanten Coefficienten, und $\tau_{0}$ eine algebraische Function bedeutet. Hieraus folgt, wenn für $u_{1}=c_{1}$ die Function $x$ in $x^{\prime}$ übergeht,

$$
\sigma_{1}\left(u_{2}, u_{3}, \cdots, u_{k}\right)=\vartheta_{0}\left(x^{\prime}, \tau_{0}\left(c_{1}\right), c_{1}, u_{2}, \cdots, u_{h}\right) .
$$

Nun ergeben die Gleichungen (19):

$$
\begin{aligned}
x^{\prime}= & \vartheta_{2}^{\prime}\left(\sigma_{2}\left(c_{1}, u_{3}, \cdots, u_{k}\right), \tau_{2}\left(u_{2}\right)\right) \\
= & \vartheta_{3}^{\prime}\left(\sigma_{3}\left(c_{1}, u_{2}, u_{4}, \cdots, u_{k}\right), \tau_{3}\left(u_{3}\right)\right) \\
& \vdots \\
= & \vartheta_{A}^{\prime}\left(\sigma_{h}\left(c_{1}, u_{2}, \cdots, u_{h-1}\right), \tau_{h}\left(u_{k}\right)\right)
\end{aligned}
$$

wenn der rationale Ausdruck $\vartheta_{v}$ für $u_{i}=c_{1}$ in $\vartheta_{v}$ "ubergeht; also lehrt die Gleichung $(22)$, dass die Function $\sigma_{1}\left(u_{2}, u_{3}, \cdots, u_{k}\right)$, wenn unter $\mu$ ein beliebiger der Werthe $2,3, \cdots, h$ verstanden wird, rational ausgedrückt werden kann dorch eine algebraische Function ron $u_{\mu}$ allein, und eine algebraische Function der Grössen $u_{2}, u_{3}, \cdots, u_{n}$ mit Ansschluss von $u_{\mu}$. Demnach besteht für die Function $\epsilon_{1}$ ein den Glei- 
chungen (19) ganz analoges Gleichungssystem. In diesen neuen Gleichungen treten algebraische Functionen von $h-2$ Variabeln auf, für welche dieselben Schlüsse wie für $\sigma_{1}$ entwickelt werden können, und durch Fortsetzung dieses Beweisverfahrens kommt man sebliesslich zu dem Resnltat,

dass eine den Gleichungen (19) genügende algebraische Function der Grössen $u_{1}, u_{2}, \cdots, u_{h}$ aus algebraischen Functionen von je einer der $h$ Grössen $u$ rational zusammengesetzt werden kann.

Hierans ergiebt sich in Verbindung mit dem Lehrsatz II der folgende

\section{Lehrsatz III.}

Fine algebraische Function $x$ der Variabeln $u_{1}, u_{2}, \cdots, u_{k}$ ist dann und nur dann durch $h$ algebraische Functionen von je einer der $h_{h}$ Variabeln u rational ausdrückbar, wenn folgende Bedingung erfullt ist. Wählt man den Index $\mu$ in der Reike der Zahlen 1,2, $\ldots, h$ beliebig, und betrachtet $x$ als algebraisehe Function von $u_{\mu}$ allein, indem man alle algebraischen Functionen von $u_{1}, u_{2}, \ldots, u_{\mu-1}, u_{\mu+1}, \cdots, u_{k}$ adjungirt, so muss die Discriminante der ursprïnglich gegebenen die Function $x$ definirenden Gleichung einen von den Grössen $u_{1}, u_{2}, \cdots$,

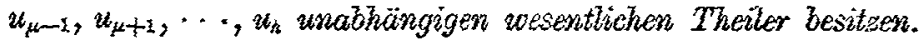

Fin ganz ähnlich lautendes Criterium für die Ausdrückbarkeit einer algebraischen Function von $h$ Variabeln durch algebraische Functionen von mehr als einer, aber weniger als $h$ Variabeln kann nach einer der hier gebrauchten ähnlichen Beweismethode abgeleitet werden; doch wird die Darstellung dieses allgemeinen Criteriums besser mit einer genaueren Untersuchung der Verzweigung der algebraischen Funetionen von mehreren Variabeln verbunden, und mag deshalb einer andern Gelegenheit vorbehalten bleiben.

Breslau, November 1886. 